\title{
Observing photonic de Broglie waves without the NOON state
}

\author{
Osung Kwon, Young-Sik Ra, and Yoon-Ho Kim* \\ Department of Physics, Pohang University of Science and Technology (POSTECH), Pohang, 790-784, Korea
}

(Dated: August 27, 2018)

\begin{abstract}
The photonic de Broglie wave, in which an ensemble of $N$ identical photons with wavelength $\lambda$ reveals $\lambda / N$ interference fringes, has been known to be a unique feature exhibited by the photon number-path entangled state or the NOON state. Here, we report the observation of the photonic de Broglie wave for a pair of photons, generated by spontaneous parametric down-conversion, that are not photon number-path entangled. We also show that the photonic de Broglie wave can even be observed for a pair of photons that are completely separable (i.e., no entanglement in all degrees of freedom) and distinguishable. The experimental and theoretical results suggest that the photonic de Broglie wave is, in fact, not related to the entanglement of the photons, rather it is related to the indistinguishable pathways established by the measurement scheme.

PACS numbers: 42.50.Dv, 42.50.-p, 42.65.Lm, 42.50.Ex
\end{abstract}

\section{INTRODUCTION}

The nature of multipartite quantum entanglement is often manifested in quantum interference experiments. For example, in the case of entangled photon states generated by spontaneous parametric down-conversion (SPDC), quantum interference is observed in coincidence counts between two detectors, each individually exhibiting no interference fringes [1-3].

One notable example of photonic quantum interference is the photonic de Broglie wave in which an ensemble of $N$ identical photons with wavelength $\lambda$ exhibits $\lambda / N$ interference fringes [4]. The photonic de Broglie wavelength $\lambda / N$ can be observed at the $N$-photon detector placed at an output port of an interferometer if the beam splitters that make up the interferometer do not randomly split $N$ photons. The quantum state of the photons in the interferometer is then the photon number-path entangled state or the NOON state

$$
|\psi\rangle=\left(|N\rangle_{1}|0\rangle_{2}+|0\rangle_{1}|N\rangle_{2}\right) / \sqrt{2}
$$

where the subscripts refer to the two interferometric paths. For this reason, the photonic de Broglie wave has been considered to be a unique feature exhibited by the NOON state and essential for quantum imaging and quantum metrology 5-7]. Experimentally, photonic de Broglie waves up to $N=4$ have been observed with corresponding NOON states [8-12].

Note, however, that $\lambda / N$ modulations in the coincidence rate among $N$ detectors may not necessarily be of quantum origin. For instance, $\lambda / N$ modulation in coincidences among $N$ detectors reported in Ref. [13], with each detector placed at an output port of a multi-path interferometer, is a classical effect since the coincidence modulation is a direct result of modulations (with different phases) observed at individual detectors. Also,

\footnotetext{
* yoonho72@gmail.com
}

classical thermal light may exhibit sub-wavelength interference fringes in coincidences but at the reduced visibility consistent with classical states [14, 15]. Thus, the reduced-period fringe itself need not be of quantum origin. It is, however, important to point out that $N$-th order quantum interference, such as quantum optical $\lambda / N$ modulations due to the photonic de Broglie wave, must exhibit high visibility (up to $100 \%$ in principle) in the absence of any lower-order interference.

In this paper, we report an intriguing new observation of $\lambda / N(N=2)$ photonic de Broglie wave interference that has no classical interpretation and is not associated with the NOON state. We also show theoretically that photonic de Broglie waves can even be observed for a pair of photons that are completely separable (i.e., no entanglement in all degrees of freedom) and distinguishable. The experimental and theoretical results suggest that the photonic de Broglie wave is, in fact, not related to the entanglement of the photons, rather it reflects the characteristics (i.e., the indistinguishable pathways) of the measurement scheme.

\section{EXPERIMENTAL OBSERVATION OF PHOTONIC DE BROGLIE WAVES WITHOUT THE NOON STATE}

Consider the experimental setup shown in Fig. 1. A $405 \mathrm{~nm}$ blue diode laser, with the full width at half maximum (FWHM) bandwidth of $0.67 \mathrm{~nm}$, pumps a $3 \mathrm{~mm}$ thick type-I BBO crystal to generate, via the SPDC process, a pair of energy-time entangled photons centered at $\lambda=810 \mathrm{~nm}$. The photon pair is coupled into the singlemode optical fiber after passing through the interference filter with a FWHM bandwidth of $5 \mathrm{~nm}$. For optimal coupling, the pump was focused at the BBO and the focal spot was imaged to the single-mode fiber [16].

The photon pair is then sent to a Mach-Zehnder interferometer (MZI), formed with BS1 and BS2, via the different input ports $a$ and $b$. The input delay $x_{1}$ between the photons is controlled by axially moving the 


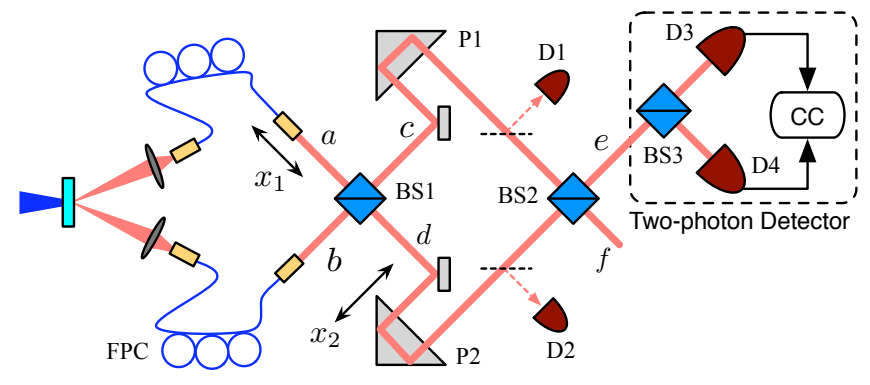

FIG. 1. Schematic of the experiment. BS1, BS2, and BS3 are 50:50 beam splitters. FPC is the fiber polarization controller and $\mathrm{CC}$ is a coincidence counter.

output collimator of a fiber and the interferometer path length difference $x_{2}$ is controlled by translating one of the trombone prisms P2. A two-photon detector, consisting of BS3 and two single-photon detectors D3 and $\mathrm{D} 4$, is placed at the output mode $e$ of MZI for photonic de Broglie wave measurement [9]. Two auxiliary detectors D1 and D2 are used to adjust the input delay $x_{1}$ by observing the Hong-Ou-Mandel (HOM) interference [17].

First, we consider the well-known photonic de Broglie wave for a biphoton NOON state and this requires preparing the state $|\psi\rangle=\left(|2\rangle_{c}|0\rangle_{d}+|0\rangle_{c}|2\rangle_{d}\right) / \sqrt{2}$ in the MZI [4, 9]. This can be accomplished by using HOM interference: the photon pair arrives at BS1 (or enters the MZI) simultaneously via the different input ports $a$ and $b$. The high-visibility HOM interference, measured in coincidence counts between D1 and D2 as a function of $x_{1}$, reported in Fig. 2 indicates that when the input delay is zero, i.e., $x_{1}=0$, the quantum state of the photons in the interferometer is indeed the desired biphoton NOON state.

Observation of the photonic de Broglie wave for the biphoton NOON state requires i) interfering the biphoton amplitudes $|2\rangle_{c}|0\rangle_{d}$ and $|0\rangle_{c}|2\rangle_{d}$ and ii) making a proper two-photon detection. In the experiment, we set $x_{1}=0$ with the help of the HOM dip in Fig. 2 and the photonic de Broglie wave corresponding to the biphoton NOON state was observed at the two-photon detector placed at the output mode $e$ of the MZI. The result shown in Fig. 3(a) exhibits $\lambda / 2$ interference fringes as a function of the MZI path length difference $x_{2}$.

We note that the coincidence between single photon detectors placed at modes $e$ and $f$ also exhibits the interference fringes with the period $\lambda / 2$ [1, 2]. This $\lambda / 2$ interference fringe, however, is, not related to the photonic de Broglie wave since i) the photons are split at BS2 and ii) it may be observed with the classical coherent state, e.g., $|0\rangle_{a}|\alpha\rangle_{b}$, at the input of the MZI [9, 13].

Consider now the situation in which the photons do not enter the MZI simultaneously. In this case, since the photons do not arrive at BS1 at the same time, HOM interference does not occur and the quantum state of the photons in the MZI is no longer the biphoton NOON state. The question we ask is whether the $\lambda / 2$ photonic

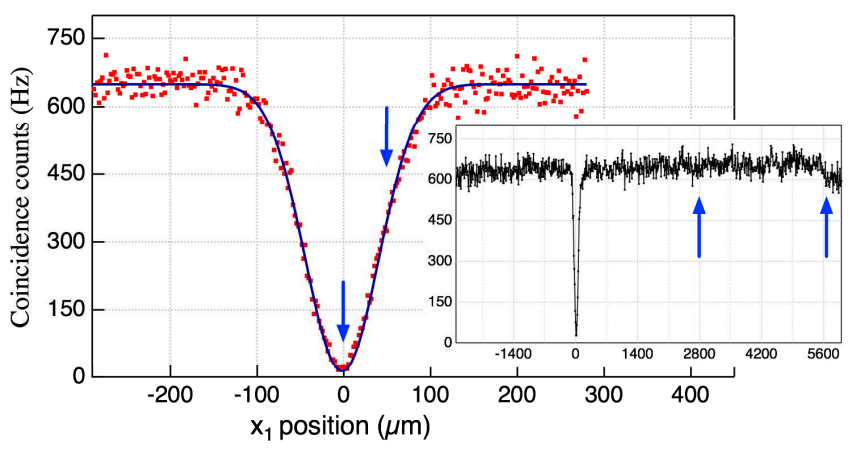

FIG. 2. The Hong-Ou-Mandel dip observed with D1 and D2. The dip visibility is better than $98 \%$. The arrows represent the $x_{1}$ positions at which the biphoton interference measurements were performed with the two-photon detector (i.e., coincidences between detectors D3 and D4).

de Broglie wave would still be observed at the two-photon detector in mode $e$ (i.e., coincidences between detectors D3 and D4) in this case.

To probe this question, we intentionally add more time delays in mode $a$ of the MZI so that $x_{1} \neq 0$. The arrows in Fig. 2 indicate the $x_{1}$ positions at which the biphoton interference measurements are made with the two-photon detector in mode $e$. First, we set $x_{1}=62 \mu \mathrm{m}$ and $x_{2}$ is scanned for the two-photon interference measurement. At this $x_{1}$ location, there is still some Hong-Ou-Mandel interference as evidenced in Fig. 2 (i.e., the coincidence rate is still below the random coincidence rate). The biphoton interference measured with the two-photon detector in this condition is shown in Fig. 3(b). Interestingly, the observed interference fringes exhibit the same $\lambda / 2$ modulation with no reduction in visibility. It is intriguing to find that the same high-visibility interference fringes with $\lambda / 2$ modulations are observed even when $x_{1}$ is completely out of the Hong-Ou-Mandel dip region. In Fig. 33(c) and Fig. 3(d), we show the biphoton interference observed with the two-photon detector at $x_{1}=2.8$ $\mathrm{mm}$ and at $x_{1}=5.7 \mathrm{~mm}$, respectively. These data correspond to the $x_{1}$ positions marked with the arrows shown in the inset of Fig. 2 .

So far, we have established experimentally that the photonic de Broglie wave can indeed be observed without the NOON state. (Note that, differently from Ref. [13], this is a real second-order quantum effect in the absence of any first-order interference: the detectors D3 and D4 individually do not show any modulations.) We now ask whether the shapes of the photonic de Broglie wave packets would remain the same. This question is probed by measuring the the photonic de Broglie wave packets for several different $x_{1}$ values and the results of these measurements are shown in Fig. [4 [18].

In Fig. 4(a), we show the typical symmetric Gaussian de Broglie wave packet for the biphoton NOON state generated by setting $x_{1}=0 \mu \mathrm{m}$. This case corresponds to Fig. 3(a). For non-NOON states (i.e., for $x_{1} \neq 0$ ), it is 

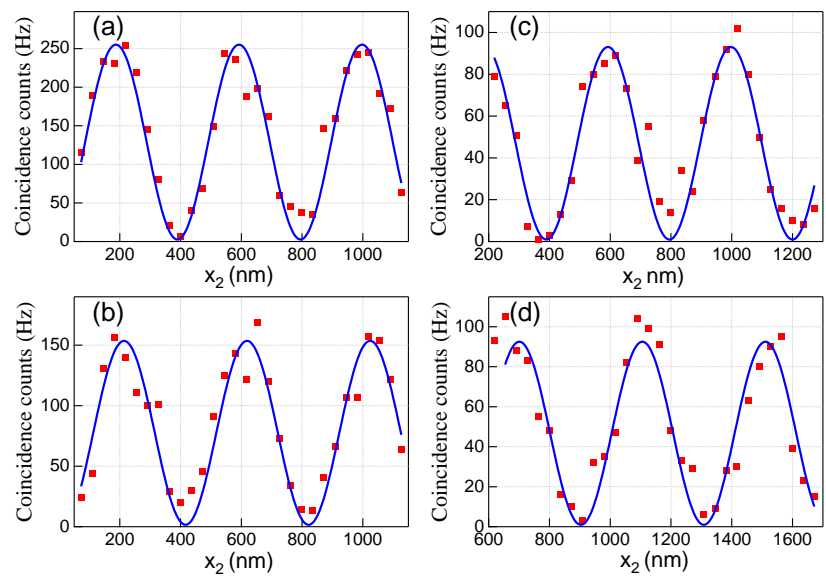

FIG. 3. Biphoton interference observed at four different $x_{1}$ positions. (a) $x_{1}=0 \mu \mathrm{m}$, (b) $x_{1}=62 \mu \mathrm{m}$, (c) $x_{1}=2.8 \mathrm{~mm}$. (a) $x_{1}=5.7 \mathrm{~mm}$. The solid lines are fit to the data with the modulation wavelength and the visibility fixed at $\lambda / 2=405$ $\mathrm{nm}$ and $98 \%$, respectively.

found that the photonic de Broglie wave packet is modified dramatically. The wave packet starts to become highly asymmetric (with respect to the random coincidence rate) as soon as $x_{1} \neq 0$, see Fig. 4 (b). The wave packet envelope then takes the shape of a double-hump and a single-dip for a larger value of $x_{1}$, see Fig. 4(c). Eventually, for a sufficiently large $x_{1}$, small side peaks starts to appear at $x_{2}= \pm x_{1}$, see Fig. 4(d). Even for very large values of $x_{1}$, e.g., corresponding to the positions marked with arrows in the inset of Fig. 2, the wave packet shape remains essentially the same as in Fig. 4(d) but the two side peaks get relocated to their new positions, $x_{2}= \pm x_{1}[18]$.

\section{PHOTONIC DE BROGLIE WAVE WITHOUT THE NOON STATE}

\section{A. Theoretical description}

To explain the observed phenomena theoretically, we start by writing the monochromatic laser pumped SPDC two-photon state as [19]

$$
|\psi\rangle_{\mathrm{e}}=\int d \omega_{s} d \omega_{i} \delta\left(\Delta_{\omega}\right) \operatorname{sinc}\left(\Delta_{k} L / 2\right) e^{i \Delta_{k} L / 2}\left|\omega_{s}, \omega_{i}\right\rangle,
$$

where the subscripts $i, s$, and $p$ refer to the idler, the signal, and the pump photon, respectively. The thickness of the SPDC crystal is $L, \Delta_{\omega}=\omega_{p}-\omega_{s}-\omega_{i}$, and $\Delta_{k}=$ $k_{p}-k_{s}-k_{i}$. Since the pump is a cw diode laser with a rather large FWHM bandwidth, the SPDC quantum state with cw diode laser pump should more properly be written as [18]

$$
\rho=\int d \omega_{p} \mathcal{S}\left(\omega_{p}\right)|\psi\rangle_{\mathrm{ee}}\langle\psi|
$$
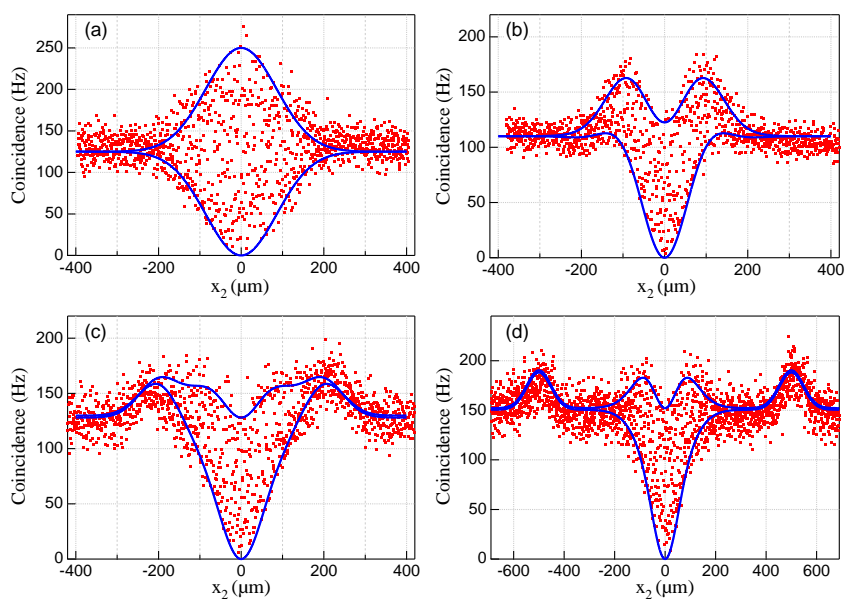

FIG. 4. The biphoton wave packet measurements with varying input delays at BS1. (a) $x_{1}=0 \mu \mathrm{m}$, (b) $x_{1}=100 \mu \mathrm{m}$, (c) $x_{1}=200 \mu \mathrm{m}$, (d) $x_{1}=500 \mu \mathrm{m}$. Within the wave packets, the modulation period is $\lambda / 2$ and the visibility around $x_{2}=0$ is better than $98 \%$. The solid lines are the wave packet envelopes calculated using Eq. (15).

where the spectral power density of the pump is assumed to be Gaussian

$$
\mathcal{S}\left(\omega_{p}\right) \equiv \exp \left(-\left(\omega_{p}-\omega_{p 0}\right)^{2} / 2 \Delta \omega_{p}^{2}\right) / \Delta \omega_{p} \sqrt{2 \pi},
$$

such that $\int \mathcal{S}\left(\omega_{p}\right) d \omega_{p}=1$.

The HOM interference can be calculated by evaluating

$$
R_{12}=\int d t d t^{\prime} \operatorname{tr}\left[\rho E_{c}^{(-)}(t) E_{d}^{(-)}\left(t^{\prime}\right) E_{d}^{(+)}\left(t^{\prime}\right) E_{c}^{(+)}(t)\right]
$$

where

$$
\begin{aligned}
& E_{c}^{(+)}(t)=\left(i E_{a}^{(+)}\left(t-\tau_{1}\right)+E_{b}^{(+)}(t)\right) / \sqrt{2} \\
& E_{d}^{(+)}(t)=\left(E_{a}^{(+)}\left(t-\tau_{1}\right)+i E_{b}^{(+)}(t)\right) / \sqrt{2},
\end{aligned}
$$

and $\tau_{1}=x_{1} / c$. The positive frequency component of the electric field in mode $a$ is given as

$$
E_{a}^{(+)}(t)=\int d \omega a(\omega) \phi(\omega) e^{-i \omega t},
$$

where $a(\omega)$ is the annihilation operator for the signal photon in mode $a$ and $E_{b}^{(+)}(t)$ for the idler photon in mode $b$ is similarly defined. The filter transmission is assumed Gaussian

$$
\phi(\omega)=\exp \left(-\left(\omega-\omega_{0}\right)^{2} / 2 \Delta \omega^{2}\right) / \sqrt{\Delta \omega \sqrt{\pi}}
$$

and $\int|\phi(\omega)|^{2} d \omega=1$. Since the natural bandwidth of $\operatorname{SPDC}, \operatorname{sinc}\left(\Delta_{k} L / 2\right)$, is much broader than the spectral filter bandwidth $\Delta \omega$, Eq. (5) is calculated to be

$$
R_{12}=1-\exp \left(-\Delta \omega^{2} \tau_{1}^{2} / 2\right) .
$$

The solid line in Fig. 2 is plotted using Eq. (10) with measured spectral filter bandwidth $\Delta \omega$. 
For the photonic de Broglie wave measurement, the response of the two-photon detector in mode $e$ must be considered and it is given as

$$
R_{e e}=\int d t d t^{\prime} \operatorname{tr}\left[\rho E_{e}^{(-)}(t) E_{e}^{(-)}\left(t^{\prime}\right) E_{e}^{(+)}\left(t^{\prime}\right) E_{e}^{(+)}(t)\right]
$$

where

$$
E_{e}^{(+)}(t)=\left(i E_{c}^{(+)}(t)+E_{d}^{(+)}\left(t-\tau_{2}\right)\right) / \sqrt{2}
$$

and $\tau_{2}=x_{2} / c$. Equation (11) can then be re-written as

$$
R_{e e}=\int d \omega_{p} \mathcal{S}\left(\omega_{p}\right) \int d t d t^{\prime}\left|\left\langle 0\left|E_{e}^{(+)}\left(t^{\prime}\right) E_{e}^{(+)}(t)\right| \psi\right\rangle_{\mathrm{e}}\right|^{2},
$$

where $\langle 0|$ denotes the vacuum state. The biphoton amplitude $\left\langle 0\left|E_{e}^{(+)}\left(t^{\prime}\right) E_{e}^{(+)}(t)\right| \psi\right\rangle_{\mathrm{e}}$ contains important information about the quantum interference and, when expanded using the electric field operators at input modes $a$ and $b$, is calculated to be

$$
\begin{aligned}
& \left\langle 0\left|E_{e}^{(+)}\left(t^{\prime}\right) E_{e}^{(+)}(t)\right| \psi\right\rangle_{\mathrm{e}}= \\
& \frac{i}{4}\left\langle 0\left|\left[\begin{array}{l}
E_{a}^{(+)}\left(t-\tau_{1}-\tau_{2}\right) E_{b}^{(+)}\left(t^{\prime}-\tau_{2}\right)+E_{a}^{(+)}\left(t^{\prime}-\tau_{1}-\tau_{2}\right) E_{b}^{(+)}\left(t-\tau_{2}\right) \\
-E_{a}^{(+)}\left(t-\tau_{1}\right) E_{b}^{(+)}\left(t^{\prime}\right)-E_{a}^{(+)}\left(t^{\prime}-\tau_{1}\right) E_{b}^{(+)}(t) \\
-E_{a}^{(+)}\left(t-\tau_{1}\right) E_{b}^{(+)}\left(t^{\prime}-\tau_{2}\right)-E_{a}^{(+)}\left(t^{\prime}-\tau_{1}\right) E_{b}^{(+)}\left(t-\tau_{2}\right) \\
+E_{a}^{(+)}\left(t-\tau_{1}-\tau_{2}\right) E_{b}^{(+)}\left(t^{\prime}\right)+E_{a}^{(+)}\left(t^{\prime}-\tau_{1}-\tau_{2}\right) E_{b}^{(+)}(t)
\end{array}\right]\right| \psi\right\rangle_{\mathrm{e}} .
\end{aligned}
$$

Note that only non-zero biphoton amplitudes are written in the above equation: terms that contain $E_{a}^{(+)} E_{a}^{(+)}$and $E_{b}^{(+)} E_{b}^{(+)}$are eventually calculated to be zero because of the nature of the input state $|\psi\rangle_{\mathrm{e}}$.

If we now consider the two-photon detector shown in Fig. 1. the normalized coincidence rate between D3 and D4 corresponds to $R_{e e}$ and is given as

$$
\begin{aligned}
R_{34}= & \frac{1}{4}\left\{4+\exp \left(-\left(\tau_{1}-\tau_{2}\right)^{2} \Delta \omega^{2} / 2\right)\right. \\
+ & \exp \left(-\left(\tau_{1}+\tau_{2}\right)^{2} \Delta \omega^{2} / 2\right)-2 \exp \left(-\tau_{2}^{2} \Delta \omega^{2} / 2\right) \\
- & 2 \cos \left(2 \omega_{0} \tau_{2}\right) \exp \left(-\tau_{2}^{2} \Delta \omega_{e}^{2} / 2\right) \\
& \left.\times\left(1+\exp \left(-\tau_{1}^{2} \Delta \omega^{2} / 2\right)\right)\right\},
\end{aligned}
$$

where $1 / \Delta \omega_{e}^{2} \equiv 1 / \Delta \omega_{p}^{2}+1 / \Delta \omega^{2}$.

Equation (15) clearly shows that the $2 \omega_{0}$ or $\lambda_{0} / 2$ interference fringe (corresponding to the photonic de Broglie wavelength), in fact, is not related to the biphoton NOON state condition $\tau_{1}=0$. As long as $\tau_{2}$ is within the effective coherence length $\sqrt{2} / \Delta \omega_{e}$, the biphoton photonic de Broglie wave interference can be observed regardless of the $\tau_{1}$ value.

Another interesting feature of Eq. (15) is that the shape of the biphoton de Broglie wave packet is $\tau_{1}$ dependent while the period of interference fringes remains the same at $2 \omega_{0}$. Note also that the maximum interference visibility is not affected by $\tau_{1}$. The theoretical result in Eq. (15) is found to be in excellent agreement with the experimental data in Fig. 3 .

\section{B. The Feynman diagram}

The interesting features of the biphoton de Broglie interference in this experiment can be intuitively understood by analyzing the Feynman diagrams representing the two-photon detection amplitudes.
Given the experimental setup in Fig. 1 there exist four Feynman paths in which the photon pair exits BS2 via the output mode $e$ and these Feynman paths are shown in Fig. 5. Since the signal, $\omega_{s}$, and idler, $\omega_{i}$, photons must always transmit/reflect or reflect/transmit at BS3 to contribute to a final two-photon detection event, each Feynman path shown in Fig. 5 branches off into two final Feynman amplitudes. There are, thus, total of eight Feynman paths which lead to a detection event at the two-photon detector in Fig. 1.

The photonic de Broglie wavelength observed in Fig. 3 is a manifestation of quantum interference among these Feynman paths. For arbitrary $\tau_{1}$ and $\tau_{2}$, the Feynman paths shown in Fig. 5 are clearly distinguishable (in time). However, if $\tau_{2}=0$, all Feynman paths become indistinguishable, regardless of $\tau_{1}$ values. This is confirmed theoretically in Eq. (15) and experimentally in Fig. 3. high-visibility $2 \omega_{0}$ or $\lambda / 2$ interference fringes are observed when $\tau_{2}$ is scanned around $\tau_{2}=0$.

In addition, it is shown in Fig. 4 that the shape of the biphoton wave packet is dependent on the $\tau_{1}$ value. In the case that $\tau_{1}=0$, the third and fourth Feynman paths in Fig. 5 5 cancel out and the wave packet envelope is determined by the overlap between the first two Feynman paths. As shown in Fig. 4(a), the result is a Gaussian wave packet whose width is determined by $\Delta \omega_{e}$.

Consider now the case of $\tau_{2}=\tau_{1}$. The signal and idler photons arrive simultaneously at BS2 for the third Feynman path in Fig. 5 and, because of the Hong-OuMandel effect, the two photons will always exit BS2 via the same output port. If we now consider the case of $\tau_{2}=$ $-\tau_{1}$, the same situation occurs for the fourth Feynman path in Fig. 5. Therefore the detection probability of the third and the fourth Feynman paths would increase twice as big compared to $\tau_{2} \neq \pm \tau_{1}$. The net results are the distinct side peaks observed at $\tau_{2}= \pm \tau_{1}$ in Fig. 4 (d).

In general, i.e., $\tau_{1} \neq 0$, all the eight Feynman paths 

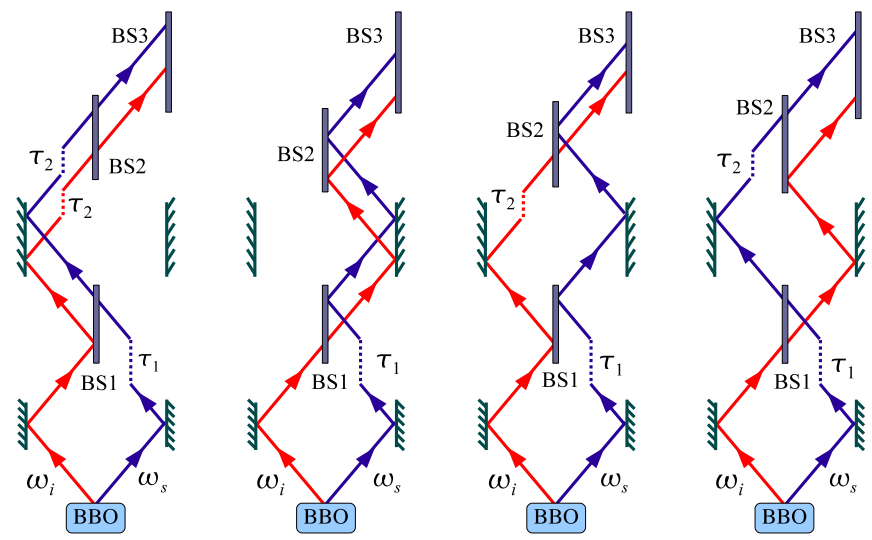

FIG. 5. The Feynman paths for the photon pair. All the Feynman paths become indistinguishable when $\tau_{2}=0$, regardless of $\tau_{1}$ values. Note that each line (top to bottom) in Eq. (14) corresponds to each Feynman path (left to right).

contribute to quantum interference in a complex manner so an intuitive explanation becomes difficult.

\section{PHOTONIC DE BROGLIE WAVE INTERFERENCE WITHOUT ENTANGLEMENT}

So far, we have shown experimentally and theoretically that the photonic de Broglie wave is in fact not related to the photon number-path entangled or the NOON state. The photonic de Broglie wave, instead, appears to be linked to the underlying spectral entanglement of SPDC photons which are used for both experimental observation and theoretical analysis [19]. The question then becomes whether the two input photons need to have any entanglement at all to exhibit the photonic de Broglie wave phenomenon.

\section{A. Photonic de Broglie wave interference for two identical photons with no entanglement}

Consider two single-photons with identical spectra and polarization, each emitted from a separate single-photon source. It is known that HOM interference can occur with a pair of identical single-photons [20, 21]. The biphoton NOON state resulting from HOM interference should then exhibit the photonic de Broglie wave.

The relevant question therefore is what would happen when there is no HOM interference between the two identical single-photons with no a priori entanglement. Would the photonic de Broglie wave still be observed in the absence of any entanglement between the photons?

To investigate this question, let us consider a singlephoton in the pure state at each input mode of the MZI in Fig. 1. Since the joint quantum state of the two singlephotons at the input modes of the MZI is separable, it can be written as

$$
|\psi\rangle_{\mathrm{s}}=\int d \omega_{a} \varphi\left(\omega_{a}\right)\left|\omega_{a}\right\rangle \otimes \int d \omega_{b} \varphi\left(\omega_{b}\right)\left|\omega_{b}\right\rangle
$$

where the single-photon spectral amplitude is assumed to be Gaussian

$$
\varphi(\omega)=\exp \left(-\left(\omega-\omega_{0}\right)^{2} / 2 \Delta \omega^{2}\right) / \sqrt{\Delta \omega \sqrt{\pi}}
$$

and $\int|\varphi(\omega)|^{2} d \omega=1$.

Given the input quantum state as in Eq. (16), the response of the MZI can now be studied. First, the singlephoton detection rates at D3 and D4 are calculated to be constant, completely independent of $x_{1}$ and $x_{2}$. This is because the single-photon detection probabilities due to the single-photons in mode $a$ and in mode $b$ have the same Gaussian envelopes but are out of phase by $180^{\circ}$. In other words, similarly to the case of entangled-photon pairs at the input, no first-order interference can be observed. Second, the two-photon detection rates for the photonic de Broglie wave measurement can be calculated by evaluating

$$
R_{e e}^{(s)}=\int d t d t^{\prime} \operatorname{tr}\left[\rho^{(s)} E_{e}^{(-)}(t) E_{e}^{(-)}\left(t^{\prime}\right) E_{e}^{(+)}\left(t^{\prime}\right) E_{e}^{(+)}(t)\right]
$$

where $\rho^{(s)}=|\psi\rangle_{\mathrm{ss}}\langle\psi|$. The above equation can then be re-written as

$$
R_{e e}^{(s)}=\int d t d t^{\prime}\left|\left\langle 0\left|E_{e}^{(+)}\left(t^{\prime}\right) E_{e}^{(+)}(t)\right| \psi\right\rangle_{\mathrm{s}}\right|^{2}
$$

The biphoton amplitude $\left\langle 0\left|E_{e}^{(+)}\left(t^{\prime}\right) E_{e}^{(+)}(t)\right| \psi\right\rangle_{\mathrm{s}} \quad$ in Eq. (19) is evaluated to be

$$
\begin{aligned}
& \left\langle 0\left|E_{e}^{(+)}\left(t^{\prime}\right) E_{e}^{(+)}(t)\right| \psi\right\rangle_{\mathrm{s}}= \\
& \frac{i}{4}\left\langle 0\left|\left[\begin{array}{l}
E_{a}^{(+)}\left(t-\tau_{1}-\tau_{2}\right) E_{b}^{(+)}\left(t^{\prime}-\tau_{2}\right)+E_{a}^{(+)}\left(t^{\prime}-\tau_{1}-\tau_{2}\right) E_{b}^{(+)}\left(t-\tau_{2}\right) \\
-E_{a}^{(+)}\left(t-\tau_{1}\right) E_{b}^{(+)}\left(t^{\prime}\right)-E_{a}^{(+)}\left(t^{\prime}-\tau_{1}\right) E_{b}^{(+)}(t) \\
-E_{a}^{(+)}\left(t-\tau_{1}\right) E_{b}^{(+)}\left(t^{\prime}-\tau_{2}\right)-E_{a}^{(+)}\left(t^{\prime}-\tau_{1}\right) E_{b}^{(+)}\left(t-\tau_{2}\right) \\
+E_{a}^{(+)}\left(t-\tau_{1}-\tau_{2}\right) E_{b}^{(+)}\left(t^{\prime}\right)+E_{a}^{(+)}\left(t^{\prime}-\tau_{1}-\tau_{2}\right) E_{b}^{(+)}(t)
\end{array}\right]\right| \psi\right\rangle_{\mathrm{s} .}
\end{aligned}
$$

Finally, the normalized coincidence rate on D3 and D4 in Fig. 1 is proportional to $R_{e e}^{(s)}$ and is given as

$$
\begin{aligned}
R_{34}^{(s)}= & \frac{1}{4}\left\{4+\exp \left(-\left(\tau_{1}-\tau_{2}\right)^{2} \Delta \omega^{2} / 2\right)\right. \\
+ & \exp \left(-\left(\tau_{1}+\tau_{2}\right)^{2} \Delta \omega^{2} / 2\right)-2 \exp \left(-\tau_{2}^{2} \Delta \omega^{2} / 2\right) \\
- & 2 \cos \left(2 \omega_{0} \tau_{2}\right) \exp \left(-\tau_{2}^{2} \Delta \omega^{2} / 2\right) \\
& \left.\times\left(1+\exp \left(-\tau_{1}^{2} \Delta \omega^{2} / 2\right)\right)\right\} .
\end{aligned}
$$


It is interesting to note that the result in Eq. (21) is identical to Eq. (15) but with $\Delta \omega_{e}$ replaced by $\Delta \omega$. Effectively, this means that SPDC pumped with a very broadband pump laser would give the identical result as that of two separable single-photon states. The theoretical results summarized in Fig. 6] show that the separable two-photon state of Eq. (16) at the input of the MZI gives nearly the same result as that of SPDC photons pumped with a laser with $2 \mathrm{~nm}$ FWHM bandwidth for both the NOON state $\left(x_{1}=0 \mu \mathrm{m}\right)$ and non-NOON state $\left(x_{1} \neq 0\right.$ $\mu \mathrm{m})$ conditions.

\section{B. Photonic de Broglie wave interference for two distinguishable (orthogonally polarized) photons with no entanglement}

In the previous section, we have seen that entanglement is in fact not necessary for observing the photonic de Broglie wave interference of two photons. It was however assumed that the two input single-photons were identical. In this section, we discuss the general case in which the two input single-photons are orthogonally polarized so that they are completely distinguishable. Note that the experimental schematic is kept the same as in Fig. 1] no polarization-information erasing polarizers are added to the setup.

For two orthogonally polarized single-photons, the joint quantum state is written as

$$
|\psi\rangle_{\text {dist }}=\int d \omega_{a} \phi\left(\omega_{a}\right)\left|\omega_{a}^{H}\right\rangle \otimes \int d \omega_{b} \phi\left(\omega_{b}\right)\left|\omega_{b}^{V}\right\rangle,
$$

where the superscripts $H$ and $V$ refer to horizontal and vertical polarization states, respectively. The counting rate at the two-photon detector, see Fig. 1] in the output mode $e$ of $\mathrm{BS} 2$ is then given as

$$
R_{e e}^{(d i s t)}=\sum_{p_{1}, p_{2} \in\{H, V\}} \int d t d t^{\prime} \operatorname{tr}\left[\rho^{(d i s t)} E_{e}^{p_{1}(-)}(t) E_{e}^{p_{2}(-)}\left(t^{\prime}\right) E_{e}^{p_{2}(+)}\left(t^{\prime}\right) E_{e}^{p_{1}(+)}(t)\right]
$$

where superscripts $p_{1}$ and $p_{2}$ denote polarizations and $\rho^{(d i s t)}=|\psi\rangle_{\text {dist dist }}\langle\psi|$. Equation (23) can then be re-written as

$$
\begin{aligned}
R_{e e}^{(d i s t)} & =\int d t d t^{\prime} \sum_{p_{1}, p_{2} \in\{H, V\}}\left|\left\langle 0\left|E_{e}^{p_{2}(+)}\left(t^{\prime}\right) E_{e}^{p_{1}(+)}(t)\right| \psi\right\rangle_{\mathrm{dist}}\right|^{2} \\
& =\int d t d t^{\prime}\left(\left|\left\langle 0\left|E_{e}^{H(+)}\left(t^{\prime}\right) E_{e}^{V(+)}(t)\right| \psi\right\rangle_{\mathrm{dist}}\right|^{2}+\left|\left\langle 0\left|E_{e}^{V(+)}\left(t^{\prime}\right) E_{e}^{H(+)}(t)\right| \psi\right\rangle_{\mathrm{dist}}\right|^{2}\right) .
\end{aligned}
$$

Note that terms that include electric field operators $E_{e}^{H(+)} E_{e}^{H(+)}$ and $E_{e}^{V(+)} E_{e}^{V(+)}$ are not shown because they eventually are calculated to be zero since the input photons are orthogonally polarized.

The biphoton amplitudes are then expanded as

$$
\begin{aligned}
& \left\langle 0\left|E_{e}^{H(+)}\left(t^{\prime}\right) E_{e}^{V(+)}(t)\right| \psi\right\rangle_{\text {dist }}= \\
& \frac{i}{4}\left\langle 0\left|\left[\begin{array}{l}
E_{a}^{H(+)}\left(t-\tau_{1}-\tau_{2}\right) E_{b}^{V(+)}\left(t^{\prime}-\tau_{2}\right)-E_{a}^{H(+)}\left(t-\tau_{1}\right) E_{b}^{V(+)}\left(t^{\prime}\right) \\
-E_{a}^{H(+)}\left(t-\tau_{1}\right) E_{b}^{V(+)}\left(t^{\prime}-\tau_{2}\right)+E_{a}^{H(+)}\left(t-\tau_{1}-\tau_{2}\right) E_{b}^{V(+)}\left(t^{\prime}\right)
\end{array}\right]\right| \psi\right\rangle_{\text {dist }},
\end{aligned}
$$

and

$$
\begin{aligned}
& \left\langle 0\left|E_{e}^{V(+)}\left(t^{\prime}\right) E_{e}^{H(+)}(t)\right| \psi\right\rangle_{\text {dist }}= \\
& \frac{i}{4}\left\langle 0\left|\left[\begin{array}{c}
E_{a}^{H(+)}\left(t^{\prime}-\tau_{1}-\tau_{2}\right) E_{b}^{V(+)}\left(t-\tau_{2}\right)-E_{a}^{H(+)}\left(t^{\prime}-\tau_{1}\right) E_{b}^{V(+)}(t) \\
-E_{a}^{H(+)}\left(t^{\prime}-\tau_{1}\right) E_{b}^{V(+)}\left(t-\tau_{2}\right)+E_{a}^{H(+)}\left(t^{\prime}-\tau_{1}-\tau_{2}\right) E_{b}^{V(+)}(t)
\end{array}\right]\right| \psi\right\rangle_{\text {dist }} .
\end{aligned}
$$

Finally, the normalized output of the two-photon detector (i.e., coincidence between D3 and D4) is calculated to be,

$$
R_{34}^{(\text {dist })}=\frac{1}{4}\left\{4-2 \exp \left(-\tau_{2}^{2} \Delta \omega^{2} / 2\right)-2 \cos \left(2 \omega_{0} \tau_{2}\right) \exp \left(-\tau_{2}^{2} \Delta \omega^{2} / 2\right)\right\} .
$$



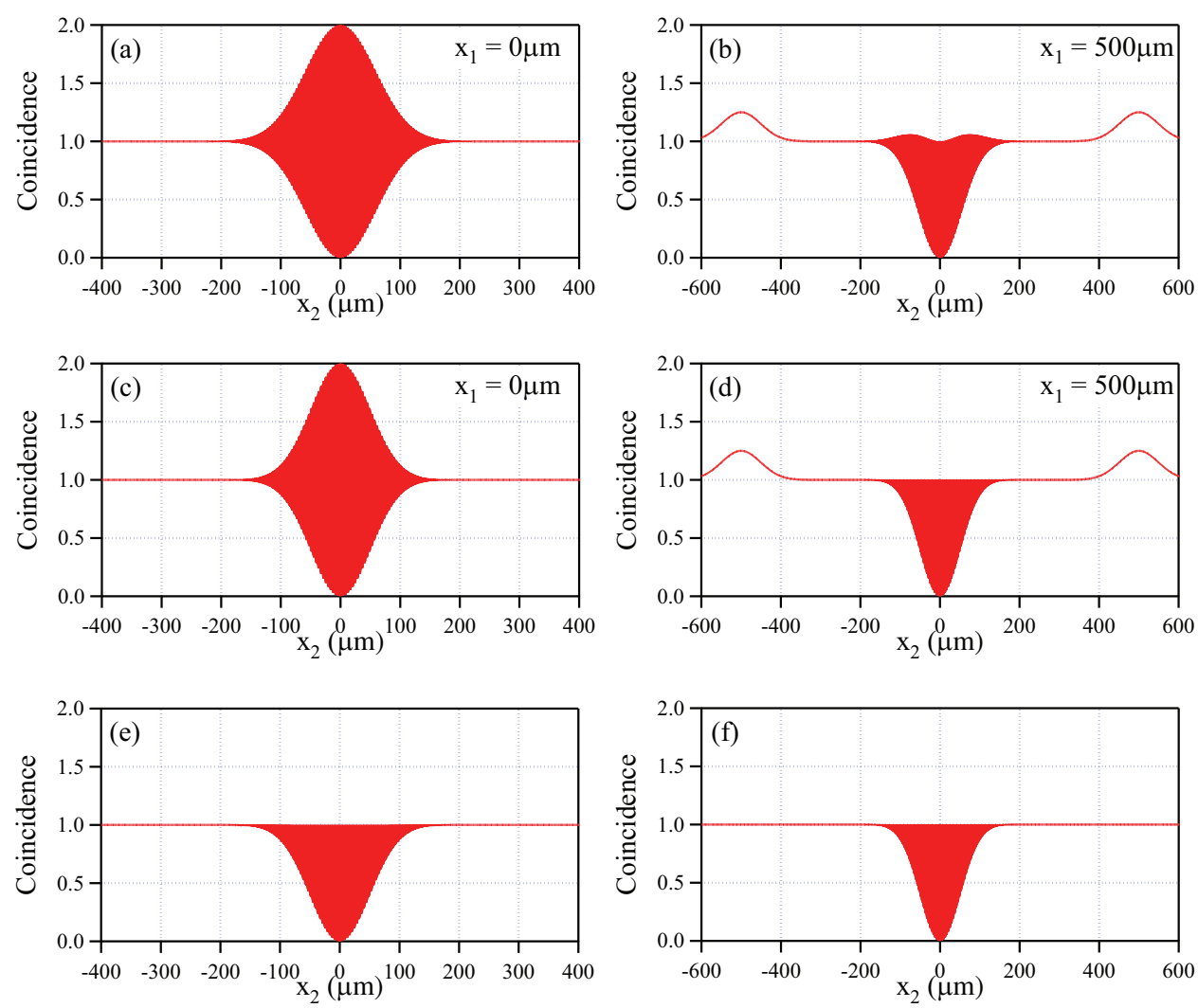

FIG. 6. Calculated photonic de Broglie wave packets for SPDC photons, (a) and (b); for two identical single-photons with no entanglement, (c) and (d); and for two distinguishable (orthogonally polarized) single-photons with no entanglement, (e) and (f). The plots (a) and (b), (c) and (d), and (e) and (f) are due to the theoretical results in Eq. (15), Eq. (21), and Eq. (27), respectively. For SPDC photons, the pump bandwidth $\Delta \omega_{p}$ is assumed to be $2 \mathrm{~nm}$ FWHM and the signal and the idler photons are filtered with $5 \mathrm{~nm}$ FWHM filters. For single-photons, they are assumed to have FWHM bandwidth of $5 \mathrm{~nm}$. Note that, since Eq. (27) is $\tau_{1}$ independent, (e) and (f) are identical plots with different ranges.

It is interesting to note that Eq. (27) also shows $2 \omega_{0}$ modulation as in the case of two identical single-photons, Eq. (21), and as in the case of a pair of SPDC photons, Eq. (15). This result, therefore, reveals that photonic de Broglie wave interference is not only unrelated to the NOON state, but it can also be observed with completely unentangled and distinguishable photons. Note also that Eq. (27) is completely independent of $\tau_{1}$ and Eq. (27) can actually be obtained from Eq. (21) by letting $\tau_{1} \rightarrow \infty$.

Equation (27) is plotted in Fig. 6(e) and Fig. 6(f). The plots show very clearly that high-visibility photonic de Broglie wave interference appear for two orthogonally polarized single-photons. Note, however, that the shape of the wave packet in Fig. 6(e) is quite different from Fig. 6(a) and Fig. 6(c) but rather similar to Fig. 6(b) and Fig. 6(d). This comes from the fact that Eq. (27) is $\tau_{1}$ independent and the other two results converge toward Eq. (27) as $\tau_{1}$ gets bigger. This fact is also reflected in the absence of side peaks in Fig. 6(f).

\section{CONCLUSION}

It is interesting to discuss the connection between the photonic de Broglie wave interference and entanglement between the input photons. The monochromaticpumped SPDC in Eq. (2) is strongly energy-time entangled and, as the pump bandwidth is increased, the degree of energy-time entanglement is reduced 22]. The experimental and theoretical results on photonic de Broglie wave interference for broadband-pumped SPDC shown in Fig. 4(d) and in Fig.6(b) make it clear that the quality of the photonic de Broglie wave interference for non-NOON states is not affected by the reduced energy-time entanglement between the photon pair. Furthermore, Fig.6(d) and Fig. 6(f) show that even two unentangled and distinguishable (orthogonally polarized) single-photons lead to essentially the same photonic de Broglie wave interference.

These results therefore reveal that entanglement between the two photons plays essentially no role in the manifestation of the photonic de Broglie wave interference. Rather, it is the measurement scheme (i.e., indis- 
tinguishable pathways established by the measurement scheme) that brings out the photonic de Broglie wave phenomenon [23].

The experimental and theoretical results in this paper apply to $N=2$ photonic de Broglie wave interference. We, however, believe that it should be possible to extend the conclusions to the $N$ photon case.

\section{ACKNOWLEDGEMENTS}

This work was supported, in part, by the National Research Foundation of Korea (KRF-2006-312-C00551,
2009-0070668, and 2009-0084473) and the Ministry of Knowledge and Economy of Korea through the Ultrafast Quantum Beam Facility Program.
[1] J. G. Rarity, P. R. Tapster, E. Jakeman, T. Larchuk, R. A. Campos, M. C. Teich, and B. E. A. Saleh, "Twophoton interference in a Mach-Zehnder interferometer," Phys. Rev. Lett.65, 1348-1351 (1990).

[2] Z. Y. Ou, X. Y. Zou, L. J. Wang, and L. Mandel, "Experiment on nonclassical fourth-order interference," Phys. Rev. A42, 2957-2965 (1990).

[3] J. Brendel, E. Mohler, and W. Martienssen, "Timeresolved dual-beam two-photon interference with high visibility," Phys. Rev. Lett.66, 1142-1145 (1991).

[4] J. Jacobson, G. Bjork, I. Chuang, and Y. Yamamoto, "Photonic de Broglie waves," Phys. Rev. Lett. 74, 48354838 (1995).

[5] A. N. Boto, P. Kok, D. S. Abrams, S. L. Braunstein, C. P. Williams, and J. P. Dowling, "Quantum Interferometric Optical Lithography: Exploiting Entanglement to Beat the Diffraction Limit," Phys. Rev. Lett.85, 2733-2736 (2000).

[6] M. D'Angelo, M. V. Chekhova, and Y. H. Shih, "Twophoton diffraction and Quatum lithography," Phys. Rev. Lett. 87, 013602 (2001).

[7] K. T. Kapale and J. P. Dowling, "Bootstrapping approach for generating maximally path-entangled photon states," Phys. Rev. Lett.99, 053602 (2007).

[8] E. J. S. Fonseca, C. H. Monken, and S. Padua, "Measurement of the de Broglie wavelength of a multiphoton wave packet," Phys. Rev. Lett.82, 2868-2871 (1999).

[9] K. Edamatsu, R. Shimizu, and T. Itoh, "Measurement of the Photonic de Broglie Wavelength of Entangled Photon Pairs Generated by Spontaneous Parametric DownConversion," Phys. Rev. Lett.89, 213601 (2002).

[10] P. Walther, J.-W. Pan, M. Aspelmeyer, R. Ursin, S. Gasparoni, and A. Zeilinger, "De Broglie wavelength of a non-local four-photon state," Nature 429, 158-161 (2004).

[11] M. W. Mitchell, J. S. Lundeen, and A. M. Steinberg, "Super-resolving phase measurements with a multiphoton entangled state," Nature 429, 161-164 (2004).

[12] T. Nagata, R. Okamoto, J. L. O'Brien, K. Sasaki, and S. Takeuchi, "Beating the Standard Quantum Limit with Four-Entangled Photons," Science 316, 726-729 (2007).
[13] K. J. Resch, K. L. Pregnell, R. Prevedel, A. Gilchrist, G. J. Pryde, J. L. O'Brien, and A. G. White, "TimeReversal and Super-Resolving Phase Measurements," Phys. Rev. Lett.98, 223601 (2007).

[14] F. Ferri, D. Magatti, A. Gatti, M. Bache, E. Brambilla, and L. A. Lugiato, "High-Resolution Ghost Image and Ghost Diffraction Experiments with Thermal Light," Phys. Rev. Lett.94, 183602 (2005).

[15] J. Xiong, D.-Z. Cao, F. Huang, H.-G. Li, X.-J. Sun, and K. Wang, "Experimental Observation of Classical Subwavelength Interference with a Pseudothermal Light Source," Phys. Rev. Lett.94, 173601 (2005).

[16] O. Kwon, Y.-W. Cho, and Y.-H. Kim, "Single-mode coupling efficiencies of type-II spontaneous parametric downconversion: Collinear, noncollinear, and beamlike phase matching," Phys. Rev. A78, 053825 (2008).

[17] C. K. Hong, Z. Y. Ou, and L. Mandel, "Measurement of subpicosecond time intervals between two photons by interference," Phys. Rev. Lett. 59, 2044-2046 (1987).

[18] O. Kwon, Y.-S. Ra, and Y.-H. Kim, "Coherence properties of spontaneous parametric down-conversion pumped by a multi-mode cw diode laser," Opt. Express 17, 1305913069 (2009).

[19] S.-Y. Baek and Y.-H. Kim, "Spectral properties of entangled photon pairs generated via frequency-degenerate type-I spontaneous parametric down-conversion," Phys. Rev. A77, 043807 (2008).

[20] C. Santori, D. Fattal, J. Vuckovic, G. S. Solomon, and Y. Yamamoto, "Indistinguishable photons from a singlephoton device," Nature 419, 594-597 (2002).

[21] P. J. Mosley, J. S. Lundeen, B. J. Smith, P. Wasylczyk, A. B. U'Ren, C. Silberhorn, and I. A. Walmsley, "Heralded Generation of Ultrafast Single Photons in Pure Quantum States," Phys. Rev. Lett. 100, 133601 (2008).

[22] Y.-H. Kim and W.P. Grice, "Measurement of the spectral properties of the two- photon state generated via type II spontaneous parametric downconversion," Opt. Lett. 30, 908-910 (2005).

[23] Y.-H. Kim and W.P. Grice, "Quantum interference with distinguishable photons through indistinguishable pathways," J. Opt. Soc. Am. B 22, 493 (2005). 